\title{
Liame
}

Liame Histoire et histoire de l'art des époques moderne et contemporaine de l'Europe méditerranéenne et de ses périphéries

26 | 2016

Collections méridionales (XVIe-XVIII ${ }^{\mathrm{e}}$ siècles)

\section{De l'Instrumentarium au Muséum. Le cabinet de Jean-François Séguier (1703-1784)}

\section{François Pugnière}

\section{OpenEdition}

\section{Journals}

\section{Electronic version}

URL: https://journals.openedition.org/liame/523

DOI: 10.4000/liame.523

ISSN: 2264-623X

\section{Publisher}

CRISES - Centre de Recherches Interdisciplinaires en Sciences Humaines et Sociales de Montpellier

\section{Electronic reference}

François Pugnière, "De l'Instrumentarium au Muséum. Le cabinet de Jean-François Séguier

(1703-1784) ", Liame [Online], 26 | 2016, Online since 02 March 2016, connection on 21 September 2021. URL: http://journals.openedition.org/liame/523 ; DOI: https://doi.org/10.4000/liame.523

This text was automatically generated on 21 September 2021.

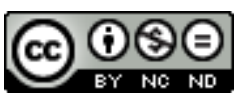

Les contenus de Liame sont mis à disposition selon les termes de la Licence Creative Commons Attribution - Pas d'Utilisation Commerciale - Pas de Modification 4.0 International. 


\title{
De l'Instrumentarium au Muséum. Le cabinet de Jean-François Séguier (1703-1784)
}

\author{
François Pugnière
}

1 Peu de cabinets méridionaux jouirent en leur temps d'une aura comparable à celui qu'avait formé Jean-François Séguier à Nîmes dans les années 1760-1770. Giacomo Casanova évoque ainsi dans ses Mémoires les «merveilles » qu'il avait pu y contempler en 1769 et qui démontraient selon lui «l'immensité de la nature et la puissance incompréhensible de celui qui l'a faite ${ }^{1} »$. Sans faire preuve de la même grandiloquence, les récits conservés abondent dans le même sens: ils attestent tous de la valeur et de l'intérêt des collections. La description laissée par Jérémie-Jacques Oberlin, en 1775, est, entre autres exemples, particulièrement évocatrice: elle s'avère une des plus substantielles et des plus enthousiastes que l'on puisse trouver dans son Journal des remarques faites dans un voyage en France ${ }^{2}$. Il est par ailleurs assez symptomatique que la Conchyliologie de Dezallier d'Argenville, dans ses éditions de 1757 et de 1780, ait accordé une place de choix aux « suites » de pétrifications du Nîmois dont la description s'étend sur près de deux pages dans la dernière édition ${ }^{3}$.

2 On trouvait pourtant à Nîmes d'autres cabinets d'importance. Les collections d'histoire naturelle du médecin Roustan et du négociant Rolant figurent honorablement dans la Conchyliologie. Le cabinet d'Antiques de l'abbé Joseph Pichony, récemment révélé par Odile Cavalier, ou celui de médailles de Pierre Boudon de Clairac, qui abritait une collection de grande valeur, n'avaient de leur côté rien d'anecdotiques ${ }^{4}$. Aucun n'égalait toutefois en réputation et en fréquentation celui de Séguier, comme le prouvent les carnets de visiteurs tenus par le savant nîmois ${ }^{5}$.

3 Ce document, publié par Emmanuelle Chapron, permet en effet de mieux prendre la mesure du renom de l'homme et de son cabinet, en indiquant les noms des 1402 personnes qui, entre 1773 et 1783, passèrent le seuil de l'hôtel Séguier 6 . À distance des principaux lieux de construction du savoir, mais bénéficiant du pouvoir d'attraction des 
antiquités nîmoises et d'une reconnaissance académique solidement établie, Séguier, dont l'aménité était notoire, avait fini par devenir dans les années 1760 une figure savante majeure de la province, rayonnant suffisamment au-delà des bornes du Languedoc et de la Provence pour attirer savants et visiteurs de marque.

L'analyse des inventaires des collections ne révèle pourtant pas au premier abord d'objets hors normes ou particulièrement rares et coûteux, à quelques exceptions près. Le statut acquis par ce cabinet et son possesseur conduit ainsi à s'interroger sur les modalités de l'élaboration et sur la nature même des collections, à la croisée des traditions intellectuelles antérieures, pleinement maîtrisées, et des grandes inflexions méthodologiques et conceptuelles qui firent évoluer les systèmes de classification des règnes de la Nature, les «théories de la terre » ou la perception même que l'on pouvait avoir d'une antiquité gréco-romaine davantage révélée que rêvée.

\section{Un cabinet au service de l'écriture savante}

5 Les recherches sur Jean-François Séguier, qui se sont multipliées depuis les années 1980, initiées par les publications de Daniel Roche et d'Elio Mosele, ont permis de renouveler en grande partie la biographie du personnage ${ }^{7}$. Jusqu'à ces travaux, Séguier n'avait guère suscité d'intérêt, en dehors d'une utilisation ponctuelle de ses grands recueils épigraphiques ${ }^{8}$. Les rares notices qui lui avaient été consacrées reposaient principalement sur une exploitation partielle de la correspondance et sur les éloges académiques qui s'étaient multipliés peu après sa disparition. Les Anecdotes sur la vie privée de M. Séguier, composées en 1785 par le notable nîmois Jean-César Vincens, furent ainsi souvent mises à contribution, bien qu'elles soient entachées d'un grand nombre d'approximations et d'erreurs factuelles'.

6 D'après le récit de Vincens, ce serait « une médaille d'Agrippa », que Séguier avait gagné «à des jeux d'écoliers à un de ses camarades de collège » qui aurait opéré "une révolution subite dans ses goûts et ses idées ", en le poussant, dès l'âge de dix ans, à rassembler ses premières médailles. Il en serait venu ultérieurement «à vendre jusqu'à ses chemises" pour acquérir les monnaies convoitées. Ayant fini par dominer ses passions, c'est la raison qui l'aurait peu après poussé à « accepter les propositions que lui faisait un particulier d'Arles de troquer ses médailles contre un certain nombre de livres choisis ». Ce dépouillement symbolique - et tout relatif en réalité - montre que la simple possession n'était déjà plus une finalité en soi, pas plus que la recherche des memorabilia qui n'anima guère le personnage tout au long de son existence. Il réalisa par ailleurs ses premiers relevés épigraphiques dans les années 1720, mettant en forme un premier recueil d'inscriptions de Narbonnaise vers 1730. C'est à cette époque qu'il acquit également ses premiers rudiments de botanique en herborisant en compagnie de son ami Pierre Baux et du médecin Jean Mathieu dans les garrigues nîmoises ${ }^{10}$. La correspondance, conservée à partir de 1728 alors que Séguier achevait ses études de droit à Montpellier, révèle que la collection, y compris à travers ses us et ses rites, allait alors de pair avec une activité intellectuelle soutenue, au point d'inquiéter un père soucieux du devenir d'un fils aîné amené à lui succéder dans sa charge de conseiller au présidial.

7 Ces craintes étaient fondées: la venue à Nîmes de Scipione Maffei, en octobre 1732, bouleversa en effet l'existence du jeune avocat au-delà de tout ce que sa famille avait pu imaginer ${ }^{11}$. Séguier, malgré les réticences des siens qui n'avaient consenti à son départ que pour quelques semaines, accompagna le marquis dans son vaste tour européen, 
résidant à Paris de janvier 1733 à mai 1736, avant de séjourner en Angleterre de mai à août 1736, puis de gagner les Provinces-Unies ${ }^{12}$ (fig. 1a et 1b). Traversant le Saint-Empire à grandes étapes pour rejoindre Vienne, où ils restèrent plus d'un mois en novembre 1736, les deux hommes rejoignirent ensuite Venise et enfin Vérone, où Séguier s'installa dans un appartement du Palazzo Maffeiano, disposant de son propre jardin botanique et de la complète jouissance de l'instrumentarium du marquis, qui reposait alors sur une modeste bibliothèque, une "suite » d'inscriptions disparates et un médailler de grande valeur ${ }^{13}$.

Figure 1a : Itinéraire de voyage et cabinets visités

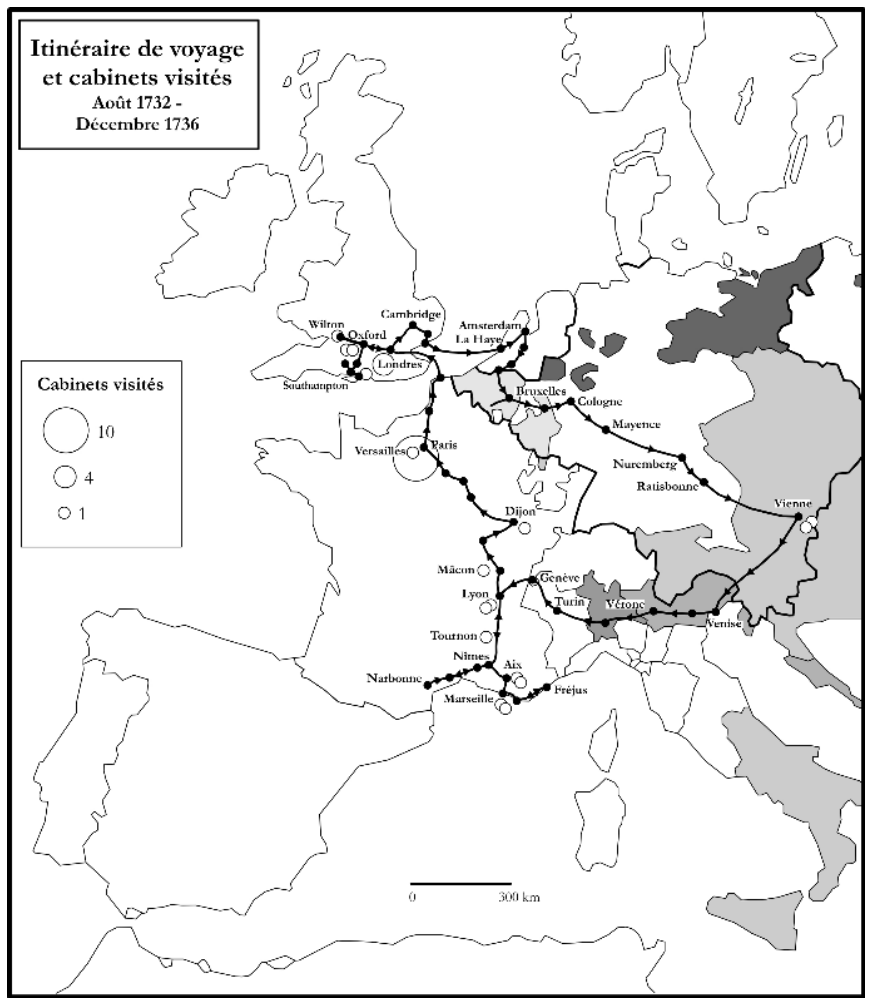


Figure 1b : Voyages en Italie et cabinets visités

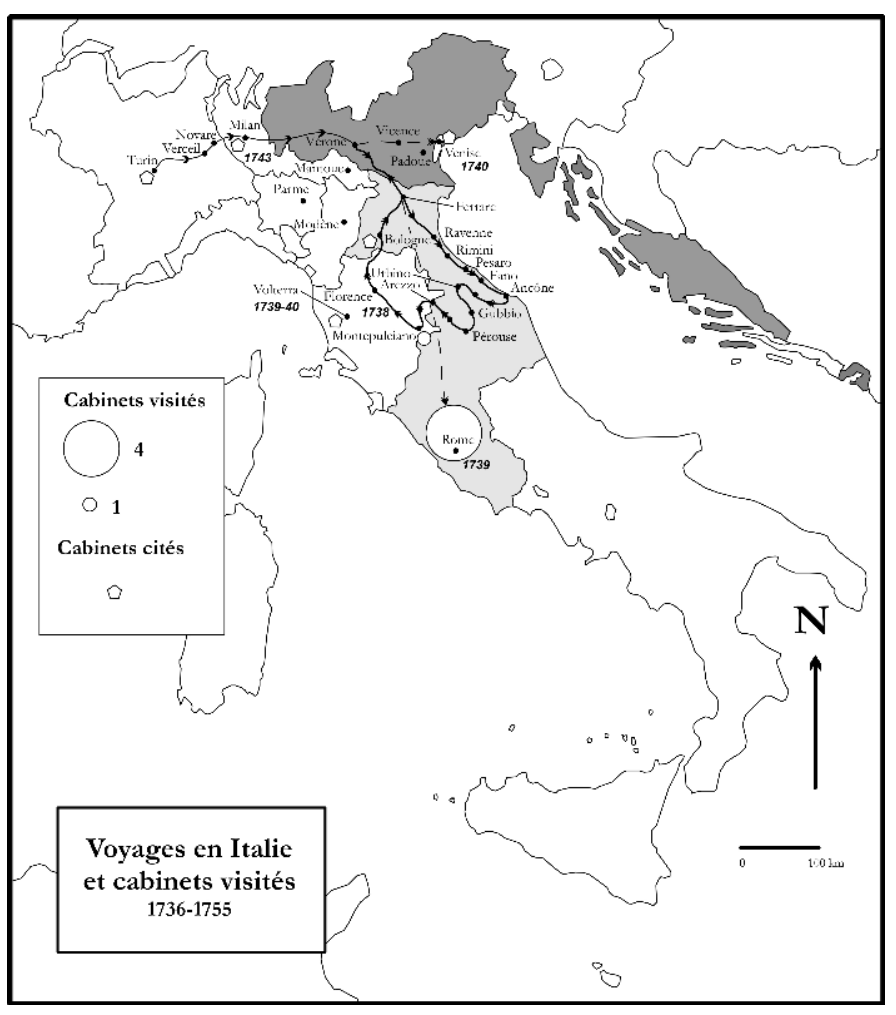

8 Le "fidèle Acate " participa dès lors activement aux grandes entreprises de son protecteur et ami, l'accompagnant en Étrurie en 1738, puis à Rome en 1739, ainsi qu'à Venise en 1741 et 1744 et en Piémont en $1743^{14}$. Maîtrisant la langue italienne avec aisance, il acquit peu à peu la reconnaissance de ses pairs, comme l'a montré Emmanuelle Chapron, en mettant en avant l'ampleur de ses affiliations académiques à partir des années $1740^{15}$. Sa proximité avec Maffei compta indubitablement dans son affirmation, mais Séguier parvint aussi à s'imposer comme botaniste et astronome à part entière, notamment à l'occasion de la publication de ses Osservazioni della Cometa en $1744^{16}$. Il sut aussi habilement jouer avec les liens privilégiés qu'il avait conservés avec la France, les Provinces-Unies ou l'Angleterre, même si les relations avec cette dernière ne s'avérèrent guère durables.

Séguier, durant son séjour parisien, avait fait porter à Nîmes une grande partie de ses spécimens et de ses livres, comptant sur son ami Pierre Baux et sur un de ses frères pour entretenir son fragile herbier ${ }^{17}$. L'installation durable au Palazzo Maffeiano le contraignit toutefois à créer un second cabinet, qu'il alimenta abondamment grâce à ses échanges et à ses inlassables excursions à travers le Vicentin, le Véronais et le long de la vallée de l'Adige. Il rassembla alors d'importantes suites de pétrifications et de spécimens minéralogiques, récoltés avec opiniâtreté entre 1733 et 1755. En 1745, dans un fragment de lettre à l'un de ses frères, il affirmait posséder « plus de mille pièces » qui composent son " recueil d'Histoire naturelle ", dont un "très grand nombre de corps pétrifiés " comprenant « 60 morceaux de bois..., plus de 100 coquillages..., une trentaine de poissons qu'on trouve dans les montagnes du Véronais... une longue suite de marbres, d'agates, de cristaux, d'albâtres et d'autres pierres plus rares $^{18}$ ». Par ailleurs, le catalogue des collections minéralogiques, qu'il dicta en mai 1784 à Jean-César Vincens, permet de préciser les provenances géographiques d'une grande partie des échantillons rassemblés ${ }^{19}$ 
(fig. 2). Une majorité d'entre eux venait du nord de la péninsule, mais la collection ne se réduisait en rien aux seuls États de Venise. Le bailli Michele Sagramoso lui offrit ainsi en 1753 un lot important d'échantillons provenant de Suède et de Russie, Franz-Ernst Brückmann lui envoyant de son côté quelques pétrifications remarquables de Basse-Saxe ${ }^{20}$. La majeure partie des naturalia fut en tout cas réunie entre 1732 et 1755 , l'année où Maffei s'éteignit. Peu d'éléments, en revanche, permettent d'appréhender l'état des collections d'antiques, probablement très réduites à cette date.

Figure 2 : Collections minéralogiques de Jean-François Séguier

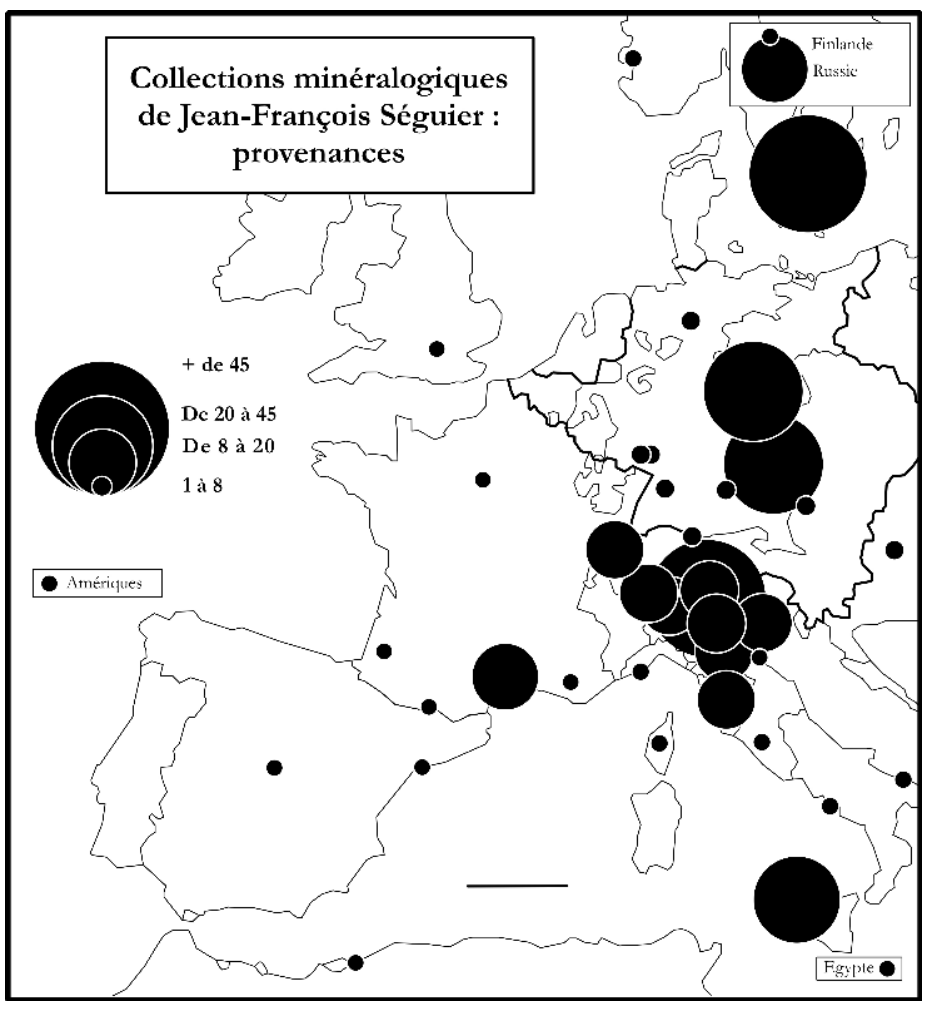

10 L'histoire de ces collections, dans leur premier état connu, permet donc de mieux comprendre leur spécificité. La connaissance des plus prestigieux cabinets européens, qu'il visita et étudia en prenant d'abondantes notes (fig. 1a et 1b), avait permis à Séguier d'acquérir une connaissance immédiate des pratiques et des usages de présentation et de conservation, au moment même où s'affirmait en Europe un véritable engouement pour les collections naturalistes ${ }^{21}$. Cette expérience influença ses propres pratiques, en ce sens où Séguier mêla étroitement le travail intellectuel et la possession de l'objet, perçu principalement à travers le concept de « suite » qui privilégiait la cohérence et la volonté d'exhaustivité. Son Catalogus plantarum, les deux premiers volumes des Plantæ Veronenses parus en 1745, ainsi que les Plantarum quæ in agro Veronensi reperiuntur imprimées en 1754, s'appuyèrent tous sur un énorme travail de collecte puis d'inventaire et de nomenclature, qui entraîna la réalisation d'un herbier spécifique de grande ampleur ${ }^{22}$. De la même manière, le travail de publication, inédit à ce jour, qu'il entreprit au début des années 1740 sur les fossiles du Monte Bolca fut élaboré à partir de l'exceptionnelle collection de pétrifications qu'il accumula et qu'il enrichit considérablement après 1751 par l'achat de la collection Spada ${ }^{23}$. La correspondance qu'il échangea à partir de 1728 avec Joseph Bimard de la Bastie révèle quant à elle l'énergie et les soins qu'il déploya pour éclairer le sens des inscriptions relevées avec minutie, mais aussi pour «expliquer » les médailles 
qu'il avait acquises ou échangées en dépassant la seule étude des "chronologies", si chères aux médaillistes ${ }^{24}$.

11 Ayant du mal à disposer des ouvrages nécessaires pour mener à bien de tels travaux, il s'efforçait alors de recourir tant bien que mal aux bibliothèques privées et donc à la prise de notes, voire au recopiage chronophage d'ouvrages in extenso. Séguier était par ailleurs un excellent dessinateur, maîtrisant avec talent le crayon, la plume et le pinceau, ce qui lui permit de constituer très tôt un remarquable « Musée de papier ${ }^{25}$ ». Essentiellement au service de la connaissance et de l'analyse, le cabinet véronais fut donc avant tout un cabinet d'étude, rien ne prouvant qu'il ait alors fait l'objet d'un effort de mise en valeur et de présentation.

\section{Collectionner pour instruire : cabinet, sociabilité et échanges savants}

Le retour de Séguier à Nîmes, après vingt-trois ans d'absence, suivit de peu la mort de Maffei en février 1755. Les biens modestes laissés par sa mère en 1754, puis par son oncle en 1755 , lui permettaient désormais de jouir d'une véritable indépendance matérielle. Le maillage complexe des réseaux qu'il avait tissés en Italie ne résista pas toutefois à l'« épreuve de la distance ", même si Séguier garda des liens de plus en plus ténus avec les milieux savants ultramontains jusqu'aux années 1770 (fig. 3a). La reconstruction des échanges épistolaires se fit désormais davantage à l'intérieur des frontières du royaume de France, plus particulièrement dans ses provinces méridionales, sans pour autant perdre l'assise européenne qu'il avait acquise à Vérone et qu'il parvint à maintenir et à élargir (fig. 3b).

Figure 3a : Correspondants de Jean-François Séguier (1728-1755)

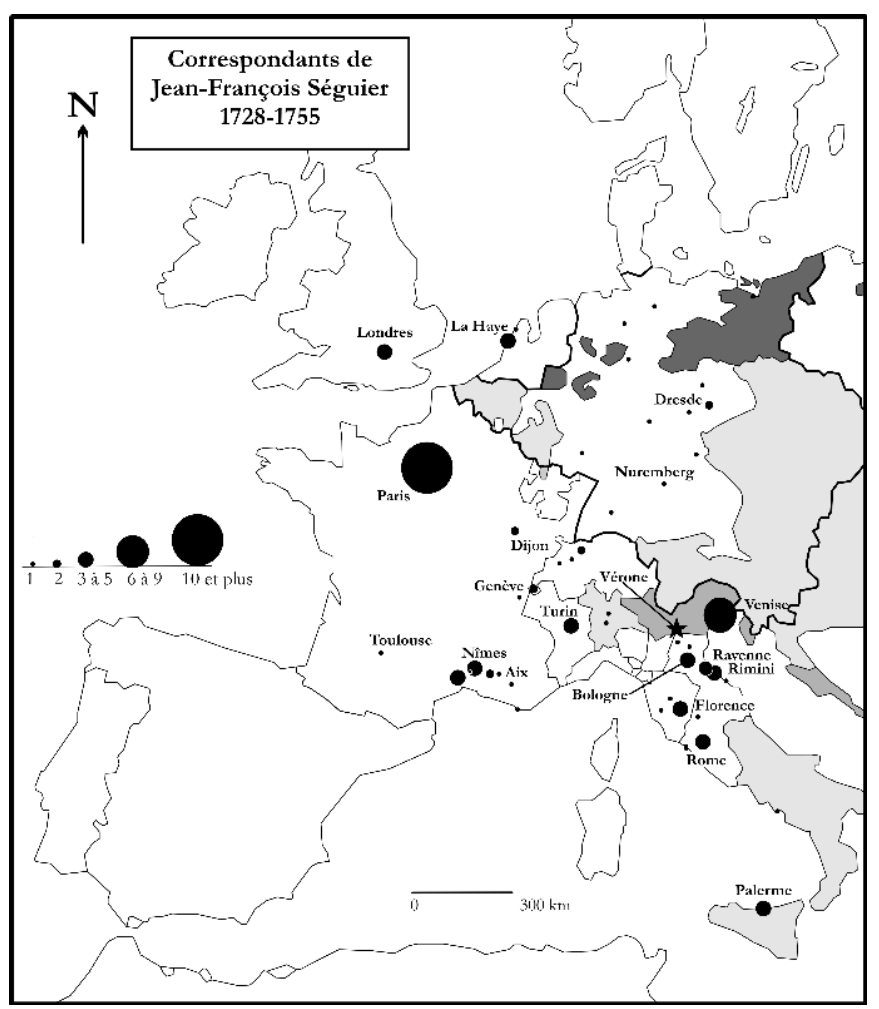




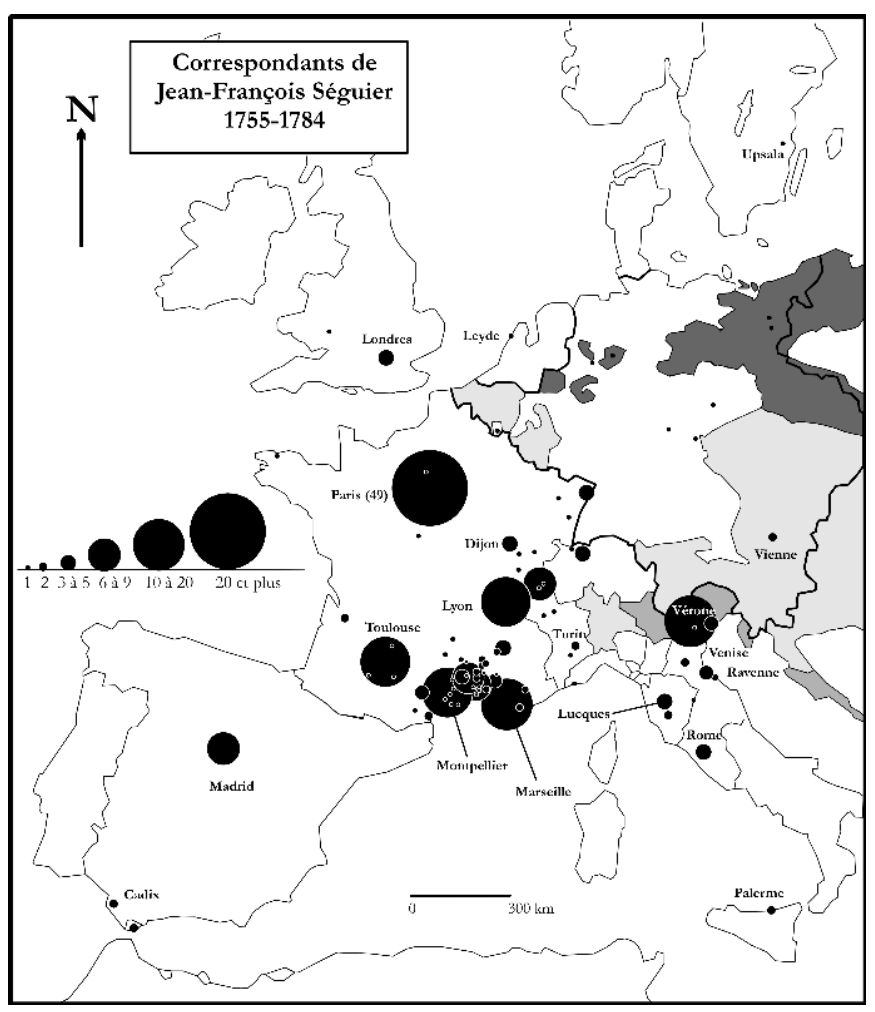

L'abbé René Séguier, le frère puiné, avait réceptionné et inventorié les vingt-et-une caisses contenant les trésors accumulés dans l'appartement loué au second étage de l'hôtel de Castelnau à Nîmes ${ }^{26}$. Ces collections furent alors réunies avec ce qui existait déjà à Nîmes et qui avait été entreposé jusqu'ici dans la maison parentale du faubourg des Calquières. Dès la fin des années 1750, malgré la relative exiguïté du nouvel appartement, les pétrifications et les échantillons minéralogiques commencèrent à être montrés aux visiteurs. L'essor de leur nombre, dès les années 1760 , contribua à la réorganisation des réseaux d'échanges et d'acquisition, en multipliant les opportunités et les offres de service. La manne offerte par les richesses du Monte Bolca avait été jusqu'ici un précieux atout, en contribuant à la construction d'une très efficace économie de l'échange entre Vérone et la France ${ }^{27}$. Séguier se rabattit opportunément sur les « ardoises arborées » que l'on trouvait à proximité de ses terres cévenoles et qu'il utilisa efficacement pour renforcer ses capacités à « obliger » en envoyant notamment de très beaux échantillons à Legouz de Gerland en 1765, à Davila en 1768 ou à Saussure en $1777^{28}$. Cette recomposition des réseaux contribua à renforcer la diversité des provenances. Ainsi, pour ne citer que quelques exemples, des échantillons de roche de la vallée du Rhin lui parvinrent via Strasbourg, complétant les spécimens ramassés in situ en 1736, de même que des « roches du lac Léman » qui lui furent envoyées par Horace-Bénédict de Saussure. Le bénédictin Salvadore Maria Blasi lui fit parvenir de son côté une importante collection de roches volcaniques de Sicile, alors que des fragments de laves du Vésuve lui furent envoyés de Naples par Lord William Hamilton en $1777^{29}$. Quant à l'élément local, dont on aurait pu soupçonner une présence plus marquée, il ne fut jamais considéré comme une catégorie distincte. Ses échantillons de roches volcaniques des environs d'Agde et de la «montagne de Sète » lui servirent ainsi à étayer une étude - inédite à ce jour - qu'il composa sur la 
structure des roches volcaniques, en les comparant aux échantillons minéralogiques des Monts d'Auvergne, de Campanie et de Sicile qu'il avait rassemblés ${ }^{30}$.

La manière dont fut élaboré et réalisé l'herbier est tout aussi caractéristique des démarches et des méthodes mises en œuvre par Séguier. Consacré dans un premier temps à l'étude de la flore languedocienne, il prit dès 1733 une tout autre ampleur. Les amitiés savantes, les rencontres nouées durant le voyage en Europe, en favorisant notamment l'extension rapide des échanges, le mirent très tôt en relation avec des savants pleinement en phase avec les grandes inflexions scientifiques que connaissait alors la botanique. Séguier, grand thuriféraire des travaux de Ray et de Tournefort, avait eu recours exclusivement à leurs systèmes lorsqu'il avait classé, à la demande de l'abbé Bignon, entre octobre 1733 et février 1734, les 33 volumes du catalogue des plantes de la Bibliothèque du roi. Il considéra dans un premier temps le système linnéen avec scepticisme - c'est du moins ce qu'il affirma à Haller et à Baux -, mais il s'intéressa peu à peu - notamment après son retour en France - à la nomenclature linnéenne, n'hésitant plus à l'utiliser conjointement avec ses systèmes de prédilection. Ce pragmatisme allié à la volonté de saisir dans sa globalité le règne végétal poussait alors Séguier à glaner tout ce qu'il était possible de rassembler. Les 144 liasses accumulées en 1784 résultaient ainsi d'une collecte systématique dans la perspective d'un "corps entier de toute l'histoire naturelle ", tel qu'il l'avait exposé à son frère en 1745 , puis à Linné et à Réaumur en $1746^{31}$ . Une telle entreprise pouvait paraître illusoire ; au moins contribua-t-elle à la réalisation d'un herbier majeur, encore en partie conservé au Musée d'histoire naturelle de Nîmes. Les collections de naturalia, si elles continuèrent à étayer le travail savant - et ce malgré l'absence de publications importantes après 1754 - revêtirent toutefois un caractère didactique et démonstratif plus marqué au fur et à mesure que les ambitions éditoriales de Séguier s'estompèrent et que les visites de son cabinet prirent de l'ampleur ${ }^{32}$.

S'il s'était illustré jusqu'ici par ses ouvrages de botanique, Séguier s'affirma en effet davantage, après 1755, en tant qu'antiquaire et numismate. La publication en 1759 de sa Dissertation sur l'inscription de la Maison Carrée ne passa pas inaperçue dans le monde savant, ni dans l'opinion éclairée, au point que Beaumarchais, sans le citer nommément, fit mention de la découverte de l'inscription dans un de ses mémoires contre Goezman en $1773^{33}$. L'Index absolutissimus, catalogue général des inscriptions latines, étrusques et grecques comportant des renvois bibliographiques systématiques, commencé en 1732, très avancé en 1755 , prit une part de plus en plus importante dans l'activité savante de l'antiquaire nîmois. Cette entreprise sans fin, qu'il ne parvint pas pourtant à conduire à terme, contribua à lui conférer une autorité scientifique incontournable dans le monde de l'épigraphie ${ }^{34}$. Considéré comme le continuateur des méthodes analytiques élaborées par Maffei, c'est lui qui mit au propre le manuscrit de l'Ars critica lapidaria, publié à Lucques par Sebastiano Donati en $1765^{35}$. Sa réception à l'Académie des Inscriptions et BellesLettres comme associé libre, en 1772, ne fit donc que couronner une réputation déjà largement assise dans la sphère érudite et savante.

La numismatique avait très tôt passionné Séguier, au point d'être considéré dès 1731 comme un bon médailliste par le président Caulet de Gragnague, numismate toulousain de renom ${ }^{36}$. Ses champs d'investigations, comme le montrent les lettres échangées avec Carl Julius Schlaeger, Joseph Pélerin ou Jules-François-Paul Fauris de Saint-Vincens, devinrent bien plus larges avec le temps et embrassèrent la totalité du monde antique, sans connaître, là encore, un recentrage particulier sur l'élément provincial. Comme l'a montré Claude Nicolet, Séguier maîtrisait de manière remarquable la «science des 
médailles ", sans nécessairement chercher à acquérir des raretés ${ }^{37}$. Il acquit ainsi en 1767 une collection de 800 pièces, mais refusa la même année une dizaine de médailles rares du Bas-Empire en raison d'un prix jugé excessif et d'un manque d'enthousiasme manifeste ${ }^{38}$. Le fait de résider à Nîmes, ville supposée riche « en antiquailles ", ne semblait pas être un atout déterminant. "Il est difficile dans nos provinces, affirmait-il ainsi au président Fauris de Saint-Vincens, de pousser certaines suites bien loin", le commerce des médailles restant trop souvent l'apanage de "quelques misérables brocanteurs ${ }^{39}$ ». Le médailler de Séguier privilégiait là encore une approche dépassant le cadre strict de la collection en s'intéressant avant tout à "l'explication». Il n'hésita pas pour cela à négliger les «occasions » en opérant un choix très directif dans ses acquisitions afin de favoriser la cohérence et l'exhaustivité.

Le savant nîmois fit preuve du même soin dans la composition et la conception de son cabinet d'antiques. La constitution de « suites " y est toutefois moins perceptible, tant la collection restait tributaire des découvertes et de la circulation plus aléatoire d'objets rares et insérés depuis le XVI siècle dans des circuits commerciaux spécifiques où les faux abondaient. La collection d'objets antiques semble en fait avoir été formée plus tardivement, après le retour à Nîmes, bien que le travail exhaustif réalisé par Michel Christol et Dominique Darde sur la collection Séguier ait mis en évidence l'existence de pièces spécifiquement italiques, comme le célèbre askos, quelques petits objets de bronzes ouvragés dont une très belle lampe, ou un énigmatique "sarcophage de Volterra $»^{40}$. Le gros du cabinet d'antiques, notamment les éléments lapidaires, fut indubitablement rassemblé après 1755 , privilégiant de facto l'élément local. Les quarante-quatre inscriptions que Séguier avait rassemblées dans son hôtel peu avant sa mort venaient en grande majorité de Nîmes et de son territoire, une partie ayant été achetée à Esprit Fléchier de Saint-Julien en $1764^{41}$. Quelques stèles, en petit nombre, lui furent par ailleurs données par des notables locaux, soit qu'ils fussent soucieux d'obliger un personnage dont la notoriété honorait le donateur, soit par pure amitié ou liens parentaux. Séguier reçut enfin en dépôt, de manière tout à fait officielle, des objets découverts à l'occasion de travaux publics, notamment des fragments de canalisation de plomb ou du mobilier de sépulture. Ainsi, en 1766, les consuls lui octroyèrent un «tuyau de congélation que l'on conservait au Temple de Diane ", et qu'il s'engageait, en signant un reçu, à représenter "à leur volontét ${ }^{42}$ ». Les objets exhumés près de l'ancien monastère de Saint-Baudile, en 1764, mais surtout en 1778, furent recueillis de la même manière. Il s'agissait d'une dizaine d'inscriptions et de divers objets funéraires pour lesquels il établit, pratique encore rare, des relevés précis et cotés, accompagnés de dessins ${ }^{43}$.

Le rapport de Séguier à l'objet antique, à quelques exceptions près où la tentation de l' unicum transparait, ne relevait donc pas, une fois encore, d'un quelconque collectionnisme. Ses conceptions se rapprochaient en fait, par maints aspects, de celles que le comte de Caylus avait exprimées dans ses écrits, et notamment dans les Recueils d'antiquités. Arguant du fait qu'il ne souhaitait pas faire « un cabinet », Caylus avait refusé en 1761 une très belle tête de Jupiter, trouvée à Nîmes, que Séguier lui avait envoyée avec une liasse de dessins et de relevés. "Je me contente, lui avait-il écrit, de rassembler des morceaux sur lesquels on peut avoir quelque opinion particulière, soit par la singularité de leurs attributs, celle de la matière ou de la nation ${ }^{44}$. » L'acquisition par Séguier en 1766 de "quelque vaisselle de cuivre qui appartenait à la cuisine des anciens" relevait visiblement d'une conception analogue. "On ne ramasse souvent dans les cabinets d'antiquaires, constatait-il dans une lettre à Joseph Pellerin, que des petites figures qu'on 
rapporte presque toujours à des divinités ». Pour Séguier, une "suite formée de tous les vaisseaux nécessaires pour le ménage et les arts utiles" s'avérait ainsi tout aussi précieux, devenant par là-même objet d'étude. Il regrettait d'ailleurs, dans la même lettre, de ne pouvoir comparer ses acquisitions avec ce qui avait été trouvé à Herculanum, faute d'avoir pu mettre la main sur un des très rares volumes des Antichità di Ercolano Esposte, publiés de manière confidentielle depuis 1757.

Séguier s'intéressa de fait, tout au long de son existence, aux aspects les plus ordinaires du monde romain, composant notamment en 1759 une Dissertation sur les cheminées des Anciens pour laquelle il s'appuya sur des objets en sa possession ou observés et dessinés par lui. Le visiteur qui parcourait son cabinet pouvait ainsi découvrir des aspects méconnus, et encore peu étudiés du monde romain que le maître des lieux se faisait un devoir d'« expliquer » avec un talent certain. Les perspectives ouvertes par les travaux de Winckelmann, l'essor du néoclassicisme et la « conversion du regard " porté sur l'objet antique n'eurent cependant guère de prise sur Séguier, qui restait fondamentalement un antiquaire : les notes qu'il tira de l'Histoire de l'art dans l'Antiquité, en 1781, se résument ainsi à huit pages d'inscriptions et de descriptions formelles ${ }^{45}$.

\section{Naissance d'un Muséum}

Le long processus qui mena à la transformation graduelle du cabinet d'étude des années véronaises en un muséum nîmois doit beaucoup à l'influence de Scipione Maffei, qui s'était très tôt intéressé aux collections lapidaires. Dès 1710, il avait prôné le recours à un lieu unique rassemblant inscriptions et objets antiques afin d'en faciliter la conservation et l'étude comparative. Il était parvenu à la conclusion que les données épigraphiques étaient beaucoup plus utiles à l'histoire que celles que l'on pouvait établir à partir des collections de médailles ${ }^{46}$. Fort de son expérience - il avait aménagé les collections royales turinoises en 1723 - et de ce qu'il avait pu observer dans les grandes collections européennes, le marquis fut amené à concevoir et à élaborer un véritable musée lapidaire, en s'inspirant de ce qui avait été mis en œuvre à Rome au Capitole en 1734. Il avait entre-temps exposé ses idées dans La religion de gentili nel morire, où il avait proposé un plan d'organisation pour que les collections de la couronne de France, et notamment les moulages de Colbert, soient mieux organisées et deviennent plus accessibles ${ }^{47}$. A Vérone, il s'appuya sur le muro delle lapidi préexistant de la cour de l'Académie philharmonique, décrit avec admiration par Montesquieu en 1728, pour développer son propre projet ${ }^{48}$. Son voyage dans la cité pontificale, en 1739, lui permit de compléter l'établissement projeté, en acquérant le plus d'inscriptions possible, quitte à sacrifier pour cela «médailles, pierres gravées, livres rares » comme l'affirmait Séguier en $1755^{49}$. Ce dernier, dont les talents de dessinateur et l'esprit méthodique s'étaient avérés précieux, comme l'a montré Lanfranco Franzoni, avait participé activement à l'entreprise, et notamment à la publication du Museum veronense en 1749, mais ce n'est qu'à la fin des années 1760 que la volonté de mettre pleinement en scène son propre cabinet vit le jour ${ }^{50}$.

En septembre 1768, il écrivit en ce sens au président Fauris de Saint-Vincens, lui annonçant qu'il venait « de construire un jardin » où il avait « placé plusieurs inscriptions antiques » qu'il voulait « destiner toutes au public » ${ }^{51}$. Il avait peu auparavant signé un bail emphytéotique avec les pères Carmes afin d'acquérir un terrain de "179 toises carrées » dans l'enclos des religieux, près du carrefour des Cinq-Vies ${ }^{52}$. Il s'agissait à cette date d'un espace extra-muros, encore peu urbanisé, malgré la croissance démographique 
que connaissait la cité. Si le jardin fût aménagé avec soin dès 1768 , les travaux de construction de la maison ne commencèrent que dans le courant de l'année $1770^{53}$. Ils étaient déjà avancés à la mi-1771, arrachant Séguier à son labeur quotidien, lui qui avait la réputation de travailler quinze heures par jour si l'on en croit Boissy d'Anglas ${ }^{54}$. Au printemps 1772, il pouvait annoncer à Carlo Allione qu'il allait bientôt transporter, « dans une maison assez grande que j'ai fait bâtir, toutes les curiosités d'histoire naturelle, les antiquités, les livres que j'ai recueillis pendant toute ma vie ${ }^{55}$ ». Il put ainsi déménager durant l'été 1772 l'essentiel de ses collections, étant à même de les montrer à ses visiteurs dès le début de l'année 1773.

Le bâtiment ayant été relativement préservé, il est aujourd'hui possible de reconstituer en partie ce que Calvet, dès 1774, ou l'abbé Bertolon, en 1778, qualifiaient expressément de muséum (fig. 4). Si le premier étage était réservé aux appartements privés, l'ensemble du rez-de-chaussée était dévolu aux collections et aux activités savantes, à quoi il fallait rajouter l'orangerie et le jardin botanique qui contenait plus d'une quarantaine d'inscriptions vers 1780 .

Figure 4 : Organisation du cabinet de Jean-François Séguier vers 1775

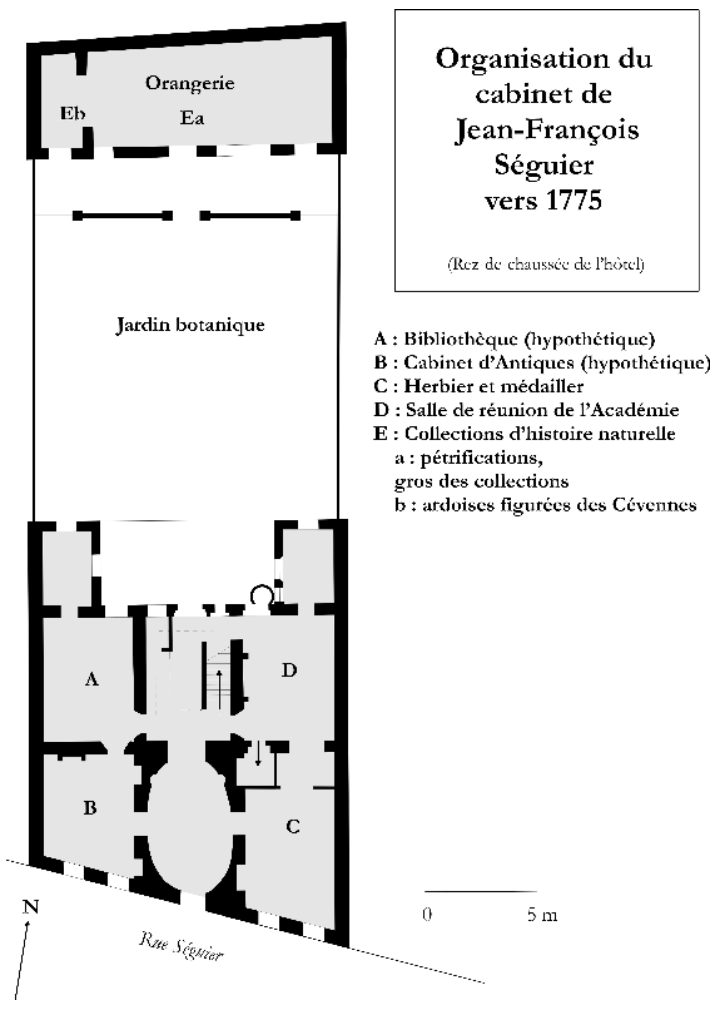

La relation laissée par Joseph Raby, en avril 1775, à l'instar de celle que rédigea Mme Cradock en 1785, montre que le cabinet d'antiques se situait dans la partie occidentale du bâtiment et communiquait directement avec la bibliothèque, qui contenait elle-même les manuscrits, les gravures et les cartes ${ }^{56}$. Il s'avère malheureusement difficile d'identifier aujourd'hui avec certitude l'affectation précise des deux pièces. De l'autre côté du vestibule, à l'Est, une première salle, dont les fenêtres donnaient sur la rue, abritait les liasses de l'herbier ainsi que le médailler et communiquait avec celle qui était utilisée comme lieu de réunion de l'Académie de Nîmes, qui donnait donc sur l'arrière de la bâtisse. Les collections d'histoire naturelle étaient quant à elles logées au fond du jardin, 
dans les deux pièces formant l'orangerie, aujourd'hui très remaniée par les ajouts ultérieurs. La plus vaste, si l'on se fie au témoignage de Raby, contenait, des «rayons simples à découvert, depuis le plafond jusqu'à trois pieds du plancher ». "Au-dessous de ces rayons, ajoutait-il, sont des tiroirs à double rang où sont les petits objets contenus dans des petits compartiments; au-dessous de ces tiroirs sont encore de plus hauts rayons pour contenir les plus gros objets ${ }^{57}$ ». C'est dans cette pièce qu'étaient présentées, savamment agencées et de manière méthodique, classées par types, les collections de pétrifications, dont les 168 «empreintes de poissons » et « d'animaux marins » du Monte Bolca et l'importante collection minéralogique (plus de 800 échantillons). La seconde pièce, plus petite, contenait essentiellement les «ardoises arborées", provenant du piémont cévenol, ainsi que les pièces les plus volumineuses qui ne pouvaient reposer sur de simples rayons.

Les montages et dispositifs en usage dans les années 1760-1770, qui ont été partiellement conservés, permettent de restituer une partie de la scénographie mise en œuvre par le maître des lieux. De petits supports en carton fort, référencés à l'aide d'une lettre et d'un chiffre renvoyant à la classification de Daubenton et sur lesquelles étaient fixés des échantillons de minéraux découpés en rectangle polis, ont notamment subsisté, de même que quelques crochets en laiton qui permettaient de fixer et de rendre plus stables les minéraux de forme irrégulière. Les échantillons de terres étaient pour leur part conservés dans des "pots» de terre, rassemblés dans une armoire vitrée. Contrairement aux dispositifs employés dans bon nombre de grands cabinets, la mise en scène des objets ne se traduisait pas systématiquement par une mise sous verre, faute probablement de moyens ${ }^{58}$. Les inventaires conservés montrent que le mobilier se composait avant tout de «tablettes", de «rayons à découvert» et de seulement cinq armoires vitrées. Le médaillier était abrité dans un meuble clos, en fort noyer, fermé par une serrure massive, et comportait toute une série de plateaux coulissants conçus à cet usage par Séguier luimême. Il s'agissait d'un meuble assez ordinaire, de facture locale ${ }^{59}$ (fig. 5). 
Figure 5 : Médailler de Jean-François Séguier

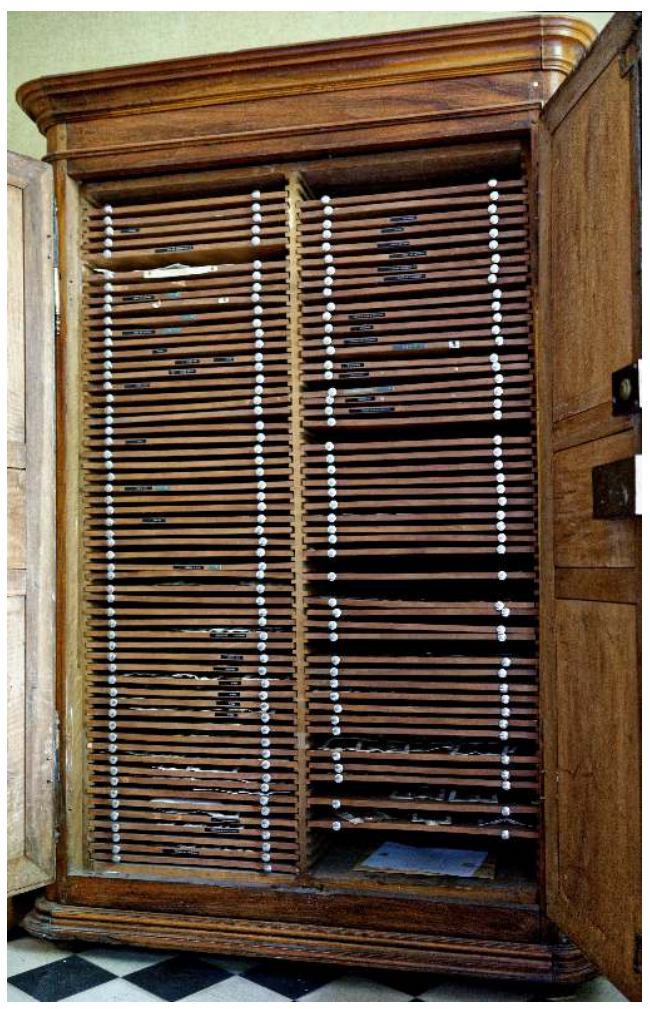

Ce cabinet n'avait par ailleurs rien d'un espace de démonstration ou d'expérimentation, tel qu'avait pu l'être celui de Réaumur à Charenton ${ }^{60}$. Il fut cependant un lieu privilégié d'échanges intellectuels et savants comme le montre le récit des trois jours passés à Nîmes par Horace-Bénédict de Saussure en $1776^{61}$. Il se rapprochait en cela du cabinet du président Bon de Saint-Hilaire, à Montpellier, qui avait toujours été « le rendez-vous des savants " selon Dezallier d'Argenville ${ }^{62}$. Séguier avait ainsi réussi à élaborer un savant équilibre entre érudition, science et sociabilité. Aussi, la découverte du cabinet se prolongeait-elle bien souvent par une visite «expliquée » des principaux monuments antiques de la ville, comme en témoignent Marmontel en 1759 ou les frères Mniszech en 1765. En pleine affirmation de la pratique du «Grand tour ", Nîmes s'était imposée comme une étape privilégiée, bien qu'excentrée, pour quiconque voulait admirer les antiquités du Midi avant le passage outre-mont ${ }^{63}$. Si l'on pouvait bénéficier du service de guides au savoir incertain, comme le déplorait Tobias Smolett en 1763, rien ne remplaçait le savoir-faire et la politesse proverbiale du célèbre antiquaire qui avait réussi à percer les mystères de l'inscription de la Maison Carrée. La visite chez « $M$. Séguier » s'insérait dans cette dynamique, au point de former un véritable continuum inséparable de la découverte des Antiques pour les visiteurs les plus notables et les plus accrédités ${ }^{64}$.

L'idée de rendre accessible au "public » ses collections, de manière pérenne, ne fit donc qu'entériner ce que l'on pourrait qualifier d'état de fait, tant le rythme des visites s'était accru. Séguier, à l'instar de Maffei, avait témoigné tout au long de son existence d'un vif intérêt pour sa ville natale et plus globalement pour la res publica, au point d'être député par le conseil de ville en 1764 pour plaider à Toulouse, avec succès, en faveur du maintien du collège de Nîmes menacé par l'expulsion des jésuites ${ }^{65}$. Il se vit confier par la suite la conduite d'un projet préalable de dégagement de l'amphithéâtre, dès 1776, et c'est lui, enfin, qui conçut et dirigea les opérations de restauration de la Maison Carrée entre 1778 
et $1781^{66}$. À ces préoccupations d'ordre civique, caractéristiques de l'esprit du temps, s'ajoutèrent les inquiétudes qu'il nourrissait quant au devenir de son cabinet, comme il l'avait ouvertement exprimé à Fauris de Saint-Vincent dès 1772, à une époque où il aspirait à une forme de « retraite littéraire ». Comme l'avaient fait un Ulisse Aldrovandi à Bologne ou un Clément de La Faille à la Rochelle, faute d'héritier susceptible de pérenniser son œuvre, il décida donc en 1778 de céder ses collections à l'Académie royale de Nîmes dont il était le secrétaire perpétuel, sous réserve qu'elles fussent rendues accessibles « au public ${ }^{67} »$. Cette donation - quatre ans avant celle de Clément de La Faille - s'inscrivait alors dans un courant, qui, à l'exemple des collections de Louis-Léon Pajot d'Ons-en-Braye, contribua à l'émergence en France de la notion de musée, défini en tant que propriété d'une personne morale, caractérisée par sa gratuité et par son ouverture régulière au public, suivant en cela le modèle italien ${ }^{68}$.

L'affaire ne fut pas toutefois des plus aisées car Séguier s'était contenté dans un premier temps de léguer ses seules collections, sans y inclure l'hôtel qui devait revenir «aux pauvres $»^{69}$. L'évêque de Nîmes, proche du savant, décida donc de racheter la bâtisse sur ses propres fonds, en versant 12000 livres à la Miséricorde et 3000 livres à l'Hôtel-Dieu. Des lettres patentes, délivrées en juillet 1779, sollicitées par Séguier et Étienne-David Meynier de Salinelles au nom de l'Académie, confirmèrent la donation qui devint totalement effective après la mort de Marianne Séguier, la dernière sœur encore en vie, en 1786. Entre-temps, le 2 décembre 1781, le conseil de ville avait donné le nom de l'illustre Nîmois au chemin qui passait devant sa bâtisse, alors que l'Académie l'élisait protecteur par « acclamation » en février $1784^{70}$.

L'hôtel, dès 1786, fut ouvert au public « le mardi, le jeudi et le samedi hors les temps de vacances selon l'institution de $M$. Séguier ", mais ni la bibliothèque, ni les collections d'histoire naturelle n'étaient encore accessibles en novembre $1789^{71}$. Les négociations conduites à partir de 1787 en vue d'installer les collections dans la Maison Carrée, qui aurait été alors échangée contre l'hôtel Séguier avec les augustins, ne purent par ailleurs aboutir, pas plus que la tentative d'impression en 1787 du manuscrit de l'Index absolutissimus, puis des Fossiles du Véronais en 1789, faute de moyens ${ }^{72}$. L'Académie avait par ailleurs du mal à assumer les frais d'entretien du cabinet et du bâtiment, ce qui avait fini par la pousser à solliciter en vain une aide financière auprès du contrôleur général des finances en 1786 puis en $1788^{73}$.

Les troubles politiques et la confiscation des collections, par la loi du 6 thermidor an II, qui suivit la suppression des Académies en 1793, sonnèrent le glas de ces projets. La maison fut vendue comme bien national, mais Aubin-Louis Millin de Grandmaison put encore relever en 1805 les inscriptions enchâssées dans les murs de l'édifice dont les derniers restes rejoignirent en 1879 seulement les collections municipales ${ }^{74}$. La plupart des objets après avoir été entreposés dans l'ancien collège, gagnèrent le Musée MarieThérèse, établi par la ville dans la Maison Carrée en 1823, le reste, notamment les naturalia, étant entreposé tant bien que mal et avec des fortunes diverses dans les magasins municipaux. Ce qui subsistait de la collection, après bien des péripéties, participa à la création des fonds des musées archéologiques et d'histoire naturelle en 1894, la bibliothèque et les manuscrits ayant de leur côté rejoint la bibliothèque publique de la ville, à l'exception des grands recueils épigraphiques confisqués par Chardon de la Rochette au profit de la bibliothèque impériale en $1805^{75}$.

Si le cabinet Séguier peut paraitre modeste, surtout quand on le compare aux grands cabinets de la seconde moitié du XVIII ${ }^{e}$ siècle, il n'en reste pas moins un bon témoin de 
l'importance prise par certaines collections provinciales dans les dernières décennies de l'Ancien Régime. Vers 1780, il s'agissait de l'un des plus importants et des plus cohérents que l'on puisse trouver en Languedoc, se caractérisant notamment par la mise en ordre systématique des collections minéralogiques et par la richesse exceptionnelle des suites de pétrifications, dont les célèbres fossiles du Monte Bolca, encore rares de ce côté des Alpes à la fin du XVIII siècle.

Comme celui de Calvet à Avignon ou du président de Robien à Rennes, pour ne citer que les mieux connus, il associait histoire naturelle et étude de l'Antiquité, fidèle en cela aux usages d'un humanisme savant dont on trouve encore trace à Nîmes sous la Monarchie de Juillet $^{76}$. Ancré dans une longue tradition érudite, le cabinet Séguier avait su préserver cet héritage en y intégrant des pratiques qui mêlaient étroitement sciences en construction, analyse comparative et mise en scène de la collection, dans un contexte de spécialisation croissante des champs disciplinaires et de remise en cause de l'érudition traditionnelle. Il reflétait en cela l'étonnante maitrise scientifique que l'homme avait fini par acquérir au prix d'une quête bibliographique constante et d'un labeur inlassable. La réputation du cabinet était en fait peu séparable de celle de l'homme.

Enfin, ces collections, en dehors du cas spécifique des objets antiques, et notamment des éléments lapidaires, ne se caractérisèrent guère par un ancrage méridional affirmé, contrairement à celles du médecin Roustan par exemple. Participant d'un projet d'étude ambitieux mais inabouti, elles s'inscrivirent davantage dans une forme de modernité européenne de la collection, façonnée tant en France qu'en Italie par une connaissance directe et peu commune des grands cabinets européens des années 1730-1740.

\section{NOTES}

1. Samuel CORDIER, Tendances et particularismes des collections provinciales au XVIIIe siècle. L'exemple du Nîmois Jean-François Séguier, thèse, Muséum National d'Histoire Naturelle, 2005, p. 412-413.

2. Jean-Jacques OBERLIN, Journal des remarques faites dans un voyage en France en 1776, Bibliothèque nationale de France, Nouvelles Acquisitions Françaises (désormais BnF, NAF) 10 040, p. 75-87.

3. Antoine-Joseph DEZALLIER D'ARGENVILLE, La Conchyliologie, ou Histoire naturelle des coquilles de mer, d'eau douce, terrestres et fossiles, avec un traité de la zoomorphose, ou représentation des animaux qui les habitent, Paris, De Bure, 1780, 2 vol., t. 1, p. 294-295.

4. Voir Odile CAVALIER, «Né pour former un cabinet comme La Fontaine pour écrire des fables. Les pagodes et marmousets du chanoine Pichony ", in François Pugnière et Véronique Krings, Nîmes et ses antiquités: un passé présent, Bordeaux, Ausonius, 2013, p. 157-168 et Odile CAVALIER, "Un ciel brillant d'images. Un recueil de dessins d'antiquités du XVIIIe siècle », in Monuments et mémoires de la fondation Eugène Piot, 92, 2013, p. 93-175.

5. Ce type de document est à vrai dire assez exceptionnel. Jean Hermann, à Strasbourg, tint de manière comparable un registre de ses visiteurs entre 1764 et 1800 (Pierre-Yves LAcour, La République naturaliste. Collections d'histoire naturelle et Révolution française, Paris, Publications scientifiques du Muséum national d'Histoire Naturelle, 2014, p. 419. L'auteur fait référence aux travaux de Dorothée Rusque). 
6. Emmanuelle CHAPRON, L'Europe à Nîmes : les carnets de Jean-François Séguier, Avignon, Barthelémy, 2008, 207 p.

7. Daniel RocHE, « Correspondants et visiteurs de Jean-François Séguier », in Elio Mosele (dir), Un accademico dei Lumi fra due città : Verona e Nîmes, Vérone, Università degli Studi di Verona, 1987, p. 33-49; Elio MOSELE, Un accademico francese del Settecento e la sua biblioteca. Jean-François Séguier, 1703-1784, Vérone, Libreria Universitaria Editrice, 1981, 106 p.; Gabriel AUDISIO et François PUGNIÈRE, Jean-François Séguier (1703-1784). Un Nîmois dans l'Europe des Lumières, Aix, Edisud, 2005, 280 p. Sa correspondance est en cours de publication : www.seguier.org.

8. Otto Hirschfeld, dans sa préface du tome XII du Corpus inscriptionum latinarum, lui rendit toutefois un hommage appuyé en 1888.

9. Anecdotes sur la vie privée de M. Séguier, lues à la séance publique de l'Académie royale de Nîmes, le 17 janvier 1785, par M. Vincens le fils, BnF, NAF 22 278, f. $509 \mathrm{r}^{\circ}-514 \mathrm{v}^{\circ}$.

10. Bibliothèque municipales de Nîmes (désormais Bibl. mun. Nîmes), ms 109.

11. Gian Paolo MARCHI, Un Italiano in Europa. Scipione Maffei tra passione antiquaria e impegno civile, Vérone, Libreria Universitaria Editrice, 1992, 285 p. et Gian Paolo ROMAGNANI (éd.), Scipione Maffei nell' Europa del Settecento, Venise, Consorzio Editori Veneti, 1998, 745 p.

12. Voir François PUGNIÈRE, Récits et carnets de voyage de Jean-François Séguier (1732-1766), à paraître en 2016. Il s'agit pour l'essentiel des manuscrits 104, 129 et 297 de la bibliothèque municipale de Nîmes.

13. Ce concept d'instrumentarium fait référence à l'ensemble des outils et des instruments mis au service de l'élaboration et de la construction des savoirs. Selon Séguier, "Son cabinet n'était composé que d'une grande suite d'inscriptions antiques [...]. Il avait un assez grand nombre de médailles antiques et quantité d'autres antiquailles [...] Son cabinet de livre n'était pas nombreux : au besoin, il recourait à ses amis qui en étaient bien pourvus, ou bien il allait passer quelque temps dans les villes qui en sont les mieux pourvues ou qui ont des bibliothèques publiques considérables... » (Bibl. mun. Nîmes, ms 134, Éloge de Maffei).

14. Expression employée par Maffei dans son testament en 1755. Pour une mise en perspective des travaux menés par Séguier en Italie voir Ivano DAL PRETE, Scienza e società nel Settecento veneto. Il caso veronese, 1680-1796, Milan, Franco Angeli, 2008, 528 p.

15. Emmanuelle CHAPRON, «Les échanges savants à l'épreuve de la distance : Jean-François Séguier (1703-1784) entre Vérone et Nîmes ", in Lucien Faggion (dir.), Du lien politique au lien social: les élites, Rives méditerranéennes, n³2-33, 2009, p. 123-139, ici p. 130 (https:// rives.revues.org/2954). Soit 15 titres académiques, dont 8 acquis pendant le séjour à Vérone.

16. Osservazioni della cometa di quest' anno 1744 e di due eclissi lunari. Fatte in Verona da Gianpaolo Guglienzi e da Gianfrancesco Seguier, Vérone, Imprimerie du séminaire, 1744, 32 p.

17. Samuel CORDIER et François PUGNIÈRE, Jean-François Séguier-Pierre Baux. Lettres. 1732-1756, Avignon, Barthelémy, 2006, $191 \mathrm{p}$.

18. Samuel CORDIER, Tendances et particularismes des collections provinciales..., op. cit., p. 186.

19. Archives départementales du Gard (désormais Arch. dép. Gard), 4 T 18.

20. Bibl. mun. Nîmes, ms 90, Pétrifications du Véronais, p. 51.

21. Aline PELLETIER, «Cabinet », in Anne Lafont (dir.), 1740. Un abrégé du monde. Savoirs et collections autour de Dezallier d'Argenville, Paris, Fage, 2012, p. 60-74.

22. Jean GAUDANT, «Les pétrifications du Véronois: un manuscrit inachevé de Jean-François Séguier (1703-1784) », Miscellanea Paleontologica $n^{\circ} 8$, Studi e ricerche sui giacimenti terziari di Bolca, 11, Vérone, 2005, p. 167-290.

23. Giovanni Giacomo Spada (1680-1747), archiprêtre de Grezzana (Pier Andrea SACCARDo, Della Storia e letteratura della flora veneta, Milan, Valentiner, 1869, p. 34-35).

24. Bibl. mun. Nîmes, ms 139. 
25. L'expression fait référence au catalogue de l'exposition Musées de papier (Elisabeth DÉculToT, Musées de papier : l'Antiquité en livres (1600-1800), Paris, Louvre éditions, 2010, 167 p.).

26. René Séguier (1705-1767) était prieur de Saint-Jean-de-Valersicle, au diocèse d'Uzès, près de la ville de Saint-Ambroix où se trouvait le gros des biens fonciers de Séguier. Il a laissé une œuvre littéraire non négligeable (Claire TORREILLES et François PUGNIÈRE, Écrire en Cévennes au XVIIIe siècle. Les œuvres de l'abbé Séguier, Montpellier, PULM, 2013, 252 p.).

27. Dans une lettre du 10 février 1754, Séguier explique à Carlo Allione avoir fait une si ample moisson des minéraux du Véronais, qu'il n'en récolte plus, sauf pour ses amis (Charles LIOTARD, « Analyse d'une collection de lettres de J.-F. Séguier à Carlo Allione de Turin ", in Mémoires de l'Académie du Gard, 1863-1864, 1865, p. 164-208, ici p. 204).

28. Emmanuelle CHAPRON, «La 'Maison des Sciences': le cabinet nîmois de Jean-François Séguier ", 2000. The European Journal, année X, 2009, $\mathrm{n}^{\circ} 1$, p. 5-6, ici p. 5 (http:// www.europeanjournal.it/dizionario_storia/european_01_2009.pdf).

29. Euvres complètes de M. le chevalier Hamilton, Paris, Moutard, 1781, p. 306.

30. Bibl. mun. Nîmes, ms 766, Mémoire sur le prisme de Saint-Tibéri.

31. Correspondance de Linné, L 0692, 3 février 1746 : « [f. $47 \mathrm{v}^{\circ}$ ] J'ai fait déjà une nombreuse collecte de livres qui traitent de toute l'histoire naturelle et j'en ramasse tous les jours. C'est pour avoir des matériaux pour un grand ouvrage que je médite. Un corps entier de toute l'histoire naturelle... C'est peut-être une idée imaginaire et qui ne s'exécutera jamais, mais quoi qu'il en soit j'aurais du moins (comme je m'en [f. $\left.48 \mathrm{r}^{\circ}\right]$ suis exprimé dernièrement en écrivant à $\mathrm{M}$. de Réaumur) une occupation qui m'occupera agréablement toute ma vie ».

32. Séguier avouait rechercher «l'occasion de parler [...] avec les étrangers qui passent par [la] ville ", se faisant "toujours un plaisir de [s]'entretenir et de [s]'instruire avec eux » (BnF, NAF 1074, f. 179 rº Jean-François Séguier à Joseph Pellerin, Nîmes, 8 octobre 1766).

33. Michel CHRISTOL (éd.), Dissertation sur l'inscription de la Maison Carrée par Jean-François Séguier, Aix, Edisud, 2005, 158 p.

34. BnF, NAF 16929 à 16935.

35. Michel CHRISTOL, « Jean-François Séguier et l'épigraphie ", Bulletin de l'École antique de Nîmes, 26, 2006, p. 3-16.

36. Bibl. mun. Nîmes, ms 141.

37. Claude NICOLET, «Le véritable projet de Jean-François Séguier ", in Alla signorina, Mélanges offerts à Noëlle de La Blanchardière, Collection de l'École française de Rome, 204, Rome, EfR, 1995, p. 311-328.

38. BnF, NAF 1074, f.187 ro Jean-François Séguier à Joseph Pellerin, 7 septembre 1767.

39. BnF, NAF 1093, f. $80 \mathrm{r}^{\circ}$ et f. $110 \mathrm{r}^{\circ}$, Jean-François Séguier à Jules-François-Paul Fauris de SaintVincens, 19 avril 1762 et 1er novembre 1779.

40. Dominique DARDE et Michel CHRISTOL, La collection Séguier au Musée archéologique, Cahiers des musées et monuments, $\mathrm{n}^{\circ}$ 12, Nîmes, Musées d'art et d'histoire de Nîmes, 2003, 93 p. Thomas Jefferson, de passage à Nîmes en 1787, en fit réaliser un modèle en bois qui servit de matrice à l'askos d'argent de Monticello House (William H. ADAMS, The Paris years of Thomas Jefferson, New Haven, Yale University Press, 2000, p. 110-112).

41. Arch. dép. Gard, $4 \mathrm{~T} 18$.

42. Archives municipales de Nîmes, 00 94, reçu délivré par le sieur Séguier, antiquaire, aux consuls de la ville, d'un « tuyau de congélation que l'on conservait au Temple de Diane », et qu'il s'engage à représenter « à leur volonté » (1766).

43. Eugène GERMER-DURAND, François GERMER-DURAND et Auguste ALLMER, Inscriptions antiques de Nîmes, Toulouse, Privat, 1893, 1100 p.

44. Bibl. mun. Nîmes, ms 141, lettre du comte de Caylus du 15 janvier 1761. 
45. Le concept de " conversion du regard" porté sur l'Antique est dû à Marc Fumaroli (Marc FUMAROLI, « Retour à l'Antique : la guerre des goûts dans l'Europe des Lumières », L'Antiquité rêvée. Innovations et résistances au XVIIIe siècle, Paris, Gallimard, 2010, p. 23-55).

46. Krzysztof POMIAN, op. cit., p. 116-117.

47. Scipione MAFFEI, La religion de gentili nel morire : ricavata da un basso rilevo antico che si conserva in Parigi, Paris, Osmont, 1736. L'ouvrage fut très violemment attaqué en France.

48. Lanfranco FRANZONI, "Origine e storia del Museo Lapidario Maffeiano », in Il Museo Maffeiano, riaperto al publico, Vérone, Comune di Verona - Direzione ai Musei, 1982, p. 29-72 et Gian Paolo MARCHI, "Scipione Maffei e il collezionismo antiquario veronese ", Est enim ille flos Italiae... vita economica e sociale nella Cisalpina romana : atti delle giornate di studi in onore di Ezio Buchi, Vérone, QuiEdit, 2008, p. 571-580.

49. Bibl. mun. Nîmes, ms 134, p. 16.

50. Lanfranco FRANZONI, «La collaborazione del Séguier alla ricerca archeologica del Maffei e suo contributo alla storia del Museo Maffeiano ", in Elio Mosele (dir), Un accademico dei Lumi fra due città..., op. cit., p. 85-100.

51. BnF, NAF 1093, f. 91-92, lettre de Séguier à Saint-Vincens du 7 septembre 1768 : « Ce sont tous des monuments trouvés dans notre ville et par conséquent ils deviennent plus intéressants pour nous ".

52. Nicole DENIS et Corinne POTAY, « La maison Séguier à Nîmes : la demeure d'un érudit dans l'îlot des Carmes », in Monuments Historiques, mai-juin 1993, n 187, p. 36-39.

53. La construction est bien connue grâce aux travaux de Christiane LASSALLE, « La maison de J.-F. Séguier ", in Jean-René Gaborit (dir.), Mécènes et collectionneurs, actes du 121e Congrès national des Sociétés Historiques et Scientifiques, Nice, 1996, tome 2 : Lyon et le Midi, Paris, CTHS, 1999, p. 121-142. 54. Les études littéraires et poétiques d'un vieillard, par le comte Boissy-d'Anglas, Paris, Kieffer, 1825, tome II, p. 277.

55. Charles LIOTARD, "Analyse d'une collection de lettres de J.-F. Séguier à Carlo Allione de Turin », Mémoires de l'Académie du Gard, année 1863-1864, 1865, p. 164-208, lettre du 15 mai 1772, p. 203.

56. Journal de Madame Cradock. Voyage en France (1783-1786), traduit d'après le manuscrit original et inédit par Mme O. DELPHIN BALleYGUIER, Paris, Perrin et Cie, 1896, 331 p.

57. Françoise WEIL, «Deux correspondants dijonnais et un visiteur grenoblois : Bouhier, Legouz de Gerland et Joseph Raby ", in Gabriel Audisio et François Pugnière, Un savant nîmois dans l'Europe des Lumières..., op. cit., p. 227-228.

58. Pierre-Yves LACoUR, La République naturaliste..., op. cit., p. 63.

59. C'est du moins ce que montre un examen attentif du meuble conservé au Musée archéologique de Nîmes.

60. Mary TERRALL, Catching Nature in the Act. Réaumur and the Practice of Natural History in the Eighteenth Century, Chicago, University of Chicago Press, 2014, p. 134-160.

61. Samuel CORDIER, Tendances et particularismes..., op. cit., p. 424-427.

62. Antoine-Joseph DEZALLIER D'ARGENVILLE, L'histoire naturelle éclaircie dans deux de ses parties principales : la lithologie et la conchyliologie, Paris, De Bure, 1742, p. 211.

63. François PUGNIÈRE, "Antiquaires et antiquités à Nîmes au siècle des Lumières ", in François Pugnière et Véronique Krings, Nîmes et ses antiquités, un passé présent, Bordeaux, Ausonius, 2013, p. 135-153.

64. Emmanuelle CHAPRON, « Du bon usage des recommandations : lettres et voyageurs au XVIIIe siècle ", in Pierre-Yves Beaurepaire et Pierrick Pourchasse (dir.), Les Circulations internationales en Europe (années 1680-années 1780), Rennes, PUR, 2010, p. 249-258.

65. Arch. dép. Gard, D 2. 
66. Bibl. mun. Nîmes, ms 304 et ms 115. Voir Gérard CAILlAT, « La Maison Carrée, histoire, usage et restaurations ", in Pierre Gros et alii, La Maison Carrée de Nîmes. Un chef-d'œuvre d'architecture romaine, Paris, Errance, 2012, p. 49-93.

67. Marie-Elisabeth BoutRoue, «Le cabinet d'Ulisse Aldrovandi et la construction du savoir », in Curiosité et cabinets de curiosités, Neuilly, Atlande, 2004, p. 43-63.

68. Krzysztof pomian. «Leçons italiennes : les musées vus par les voyageurs français au XVIIIe siècle ", in Les Musées en Europe à la veille de l'ouverture du Louvre, Paris, Klincksieck, 1995, p. 335-361.

69. Charles LIOTARD, «Donations de Séguier à l'Académie de Nîmes avec la participation de Mgr de Becedelièvre, documents inédits ", Mémoires de l'Académie de Nîmes, VIII, 1885, p. 299-325. L'auteur s'appuie sur le «Registre des arrêtés faits par le comité de l'Académie nommé par délibération du 11 septembre $1778 »$. Ce registre de 142 pages contient toute la correspondance relative à l'affaire.

70. L'Académie de Nîmes (1682-1982), documents réunis à l'occasion du tricentenaire de sa fondation, Nîmes, Musée des beaux-arts, 1982, non paginé (120p.).

71. Christiane LASSALLE, «La maison de J.-F. Séguier », art. cit., p. 140, d'après le Calendrier de la ville de Nismes et de sa sénéchaussée pour l'année 1789, Nîmes, Belle, 1789, p. 112-113.

72. Fonds Vincens-Devillas, Musée du Vieux Nîmes, vol. 1 (non coté).

73. Samuel CORDIER, Tendances et particularismes des collections provinciales..., op. cit., p. 176.

74. Aubin-Louis millin De GRANDMAISON, Voyage dans les départements du sud de la France, Paris, Imprimerie impériale, 1811, tome IV, vol. 1, p. 266-279.

75. Lettre de M. Chardon-La Rochette à A.-L. Millin sur les manuscrits de J.-Fr. Séguier, Paris, 1806 , in- $4^{\circ}$ (La lettre avait été publiée pour la première fois dans la livraison de décembre $1805 \mathrm{du}$ Magasin Encyclopédique).

76. Laurence BRocKLISS, Calvet's Web. Enlightenment and the Republic of Letters in Eighteenth-Century France, Oxford, OUP, 2002, 471 p. et Gauthier AUBERT, «Un collectionneur provincial vu par ses contemporains : le président de Robien (1698-1756) », in Annales de Bretagne et des pays de l'Ouest, vol. 105, 1998, p. 37-57.

\section{ABSTRACTS}

Jean-François Séguier's cabinet is probably one of those which, in the years 1760-1770, enjoyed the most flattering fame in the Southern part of the Kingdom, although it was not characterised by a real French southern anchoring. Cabinet of study, feeding the intellectual production during the Veronese stay of Séguier with Scipione Maffei, it took then a didactic and demonstrative dimension after Seguier went back to Nîmes in 1755. In 1778 the donation of collections to the Royal Academy of Nîmes confirmed the constitution of a true museum, shaped by a long experiment of the collection and fed by the correspondence and by the direct knowledge of great European cabinets. Rooted in a plan inherited from a tradition of learned humanism, the collections gathered naturalia and antiqua in the same place. The study of this cabinet brings to understand the mechanisms and the purpose of collection through the complex and prominent character of one of the most outstanding figures of provincial erudition. 
Le cabinet de Jean-François Séguier est probablement un de ceux qui, dans les années 1760-1770, jouirent de la renommée la plus flatteuse dans la partie méridionale du Royaume, bien qu'il ne se caractérisât guère par une méridionalité affirmée dans sa composition. D'abord cabinet d'étude, alimentant la production intellectuelle lors du séjour véronais de Séguier auprès de Scipione Maffei, il revêtit par la suite une dimension didactique et démonstrative lors du retour à Nîmes en 1755. La donation des collections à l'Académie royale de Nîmes en 1778 ne fit qu'entériner la constitution d'un muséum à part entière, façonnée par une longue expérience de la collection nourrie par l'échange épistolaire et par une connaissance peu commune et directe des grands cabinets européens. Rassemblant, selon un schéma hérité de l'humanisme savant, naturalia et antiqua, en un même lieu, l'étude ce cabinet permet de fait d'interroger les ressorts et les finalités de la collection à travers la figure complexe d'une personnalité incontournable de l'érudition provinciale.

\section{AUTHOR}

\section{FRANÇOIS PUGNIÈRE}

CRISES (EA 4424), Université Paul-Valéry, Montpellier

francois.pugniere@ac-montpellier.fr 